Forum 2020 · 35:59

https://doi.org/10.1007/s12312-019-00741-7

Online publiziert: 8. Januar 2020

(c) Springer Medizin Verlag GmbH, ein Teil von

Springer Nature 2019
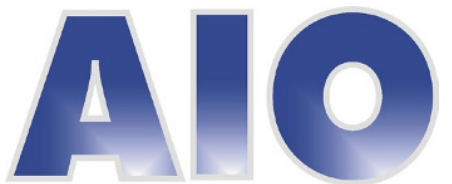

Die wissenschaftliche Arbeit „Sequential Versus Combination Therapy of Metastatic Colorectal Cancer Using Fluoropyrimidines, Irinotecan, and Bevacizumab: A Randomized, Controlled Study - XELAVIRI (AIO-KRK-0110)“, mit der sich PD Dr. Dominik Paul Modest beworben hatte (im Journal Clinical Oncology 2019 Jan 1; 37(1):22-32 veröffentlicht), wurde mit dem klinischen Teil des AIO-Wissenschaftspreises 2019 ausgezeichnet.

Die Preisverleihung erfolgte im Rahmen des 16. AIO-Herbstkongresses im November 2019 in Berlin.

Als Jurymitglieder fungierten in diesem Jahr Prof. Andreas Hochhaus, Prof. Ulrich Keilholz, Prof. Florian Lordick, Prof. Thomas Seufferlein und Prof. Jens Siveke, denen auf diesem Weg unser herzlichster Dank ausgesprochen wird! Die Laudatio hielt Prof. Michael Geißler aus Esslingen. Auch ihm gilt unser Dank!

Die ersten Ergebnisse der XELAVIRIStudie wurden bereits 2017 beim WCGICund ESMO-Kongress jeweils im Rahmen einer oral presentation vorgestellt.

Die XELAVIRI Studie ist eine randomisierte Phase III Studie, in der insgesamt 421 auswertbare Patienten (medianes Alter ca. 70 Jahre) behandelt wurden, die ein nicht resektables metastasiertes Kolorektalkarzinom hatten. Die Studie untersuchte die Nicht-Unterlegenheit einer sequenziellen Therapiestrategie (mit initialem Fluoropyrimidin plus Bevacizumab und sekundärer Eskalation unter Zugabe von Irinotecan) versus eine upfront Kombination der drei Medikamente (Fluoropyrimidin, Irinotecan, Bevacizumab). Der primäre Endpunkt war "time to failure of strategy“ mit einer Grenze bezüglich der anzunehmenden

Arbeitsgemeinschaft Internistische Onkologie e.V. (AIO)

Berlin, Deutschland

\title{
AlO-Wissenschaftspreis 2019
}

Nichtunterlegenheit von einer hazard ratio von 0,8 . Dieser Endpunkt wurde knapp verfehlt. Interessant waren innerhalb der Studienpopulation die signifikante Interaktion des RAS-Status' mit dem Endpunkt (präspezifizierte, aber nicht gepowerte Subgruppenanalyse): während bei Patienten mit RAS Wildtyp Tumor eine initiale Dreifachtherapie klar besser erschien, war das sequentielle Vorgehen bei Patienten mit einem RASmutierten Tumor der Dreifachkombination bezüglich des primären Endpunktes nicht unterlegen. Ähnliche Signale wurden konsistent auch im Gesamtüberleben verzeichnet.

Die XELAVIRI-Studie wurde als „investigator-initiated trial“ innerhalb der AIO unter Leitung der FIRE-Studiengruppe (LKP Prof. Dr. Volker Heine- mann) als nationales Projekt in insgesamt 82 Zentren rekrutiert und finanziell durch die Roche Pharma AG unterstützt. Die Daten der Studie können Therapieentscheidungen, insbesondere bei älteren Patienten, in der Erstlinientherapie des metastasiertes Kolorektalkarzinoms in Abhängigkeit des RAS-Status des Tumors unterstützen.

\section{Korrespondenzadresse}

\section{PD Dr. Dominik Paul Modest}

CharitéCentrum für Tumormedizin,

Med. Klinik m. S. Hämatologie und Onkologie und Tumorimmunologie, Charité - Universitätsmedizin Berlin

Augustenburger Platz 1, 13353 Berlin,

Deutschland

modest@aio-portal.de

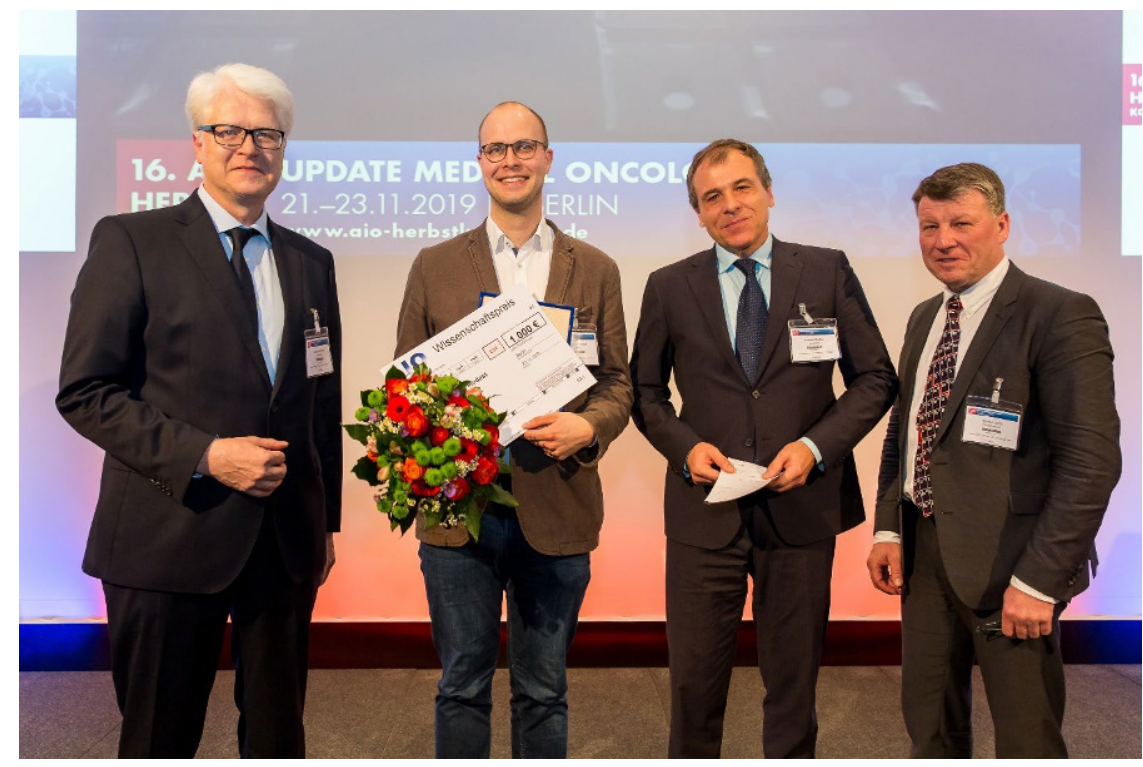

$\Delta$ v. I. n. r. Prof. Volker Heinemann, PD Dr. Dominik Paul Modest, Prof. Michael Geißler, Prof. Helmut Oettle. (@ Frederic Schweizer, Berlin) 